2007-8

\title{
Obstacle Avoidance Using Circular Paths
}

Timothy McLain

Mechanical Engineering Department, Brigham Young University, mclain@byu.edu

Randal W. Beard

Department of Electrical and Computer Engineering, Brigham Young University, beard@ee.byu.edu Jeffery Brian Saunders

Department of Electrical and Computer Engineering, Brigham Young University

Follow this and additional works at: https://scholarsarchive.byu.edu/facpub

Part of the Mechanical Engineering Commons

\section{Original Publication Citation}

Saunders, J., Beard, R., and McLain, T. Obstacle Avoidance Using Circular Paths, Proceedings of the AIAA Guidance, Navigation, and Control Conference, AIAA-2007-6604, August 2007, Hilton Head, South Carolina.

\section{BYU ScholarsArchive Citation}

McLain, Timothy; Beard, Randal W.; and Saunders, Jeffery Brian, "Obstacle Avoidance Using Circular Paths" (2007). Faculty Publications. 1913.

https://scholarsarchive.byu.edu/facpub/1913

This Conference Paper is brought to you for free and open access by BYU ScholarsArchive. It has been accepted for inclusion in Faculty Publications by an authorized administrator of BYU ScholarsArchive. For more information, please contact ellen_amatangelo@byu.edu. 


\title{
Obstacle Avoidance Using Circular Paths
}

\author{
Jeffery Saunders *and Randal Beard †and Tim McLain ${ }^{\ddagger}$ \\ Brigham Young University, Provo, Utah, 84602
}

\begin{abstract}
This paper develops a method of obstacle avoidance for fixed-wing miniature air vehicles (MAV) using a series of circular oscillating paths and a single point laser ranger. The laser ranger is a low power, light-weight device used to report the distance to an object in a single direction of the body frame of a MAV. The oscillating paths allow the laser ranger to scan for obstacles and possible escape paths for the MAV in the case of obstacle detection. The circular paths are generated along waypoint paths and transition between waypoint paths without loss of scanning capabilities. Obstacle avoidance is guaranteed.
\end{abstract}

\section{Nomenclature}

$V \quad$ Velocity

$V_{c} \quad$ Commanded Velocity

$\phi \quad$ Roll Angle

$\phi_{c} \quad$ Commanded Roll Angle

$\phi_{d} \quad$ Desired roll angle

$\psi \quad$ Heading

$\alpha_{V} \quad$ Commanded velocity approach constant

$\alpha_{\phi} \quad$ Commanded roll angle approach constant

$\psi_{d} \quad$ Heading of waypoint path

$\psi_{m} \quad$ Desired maximum deviation angle from waypoint path in circular paths

$n \quad$ Number of circles counted from current position

$R \quad$ Desired turning radius

$L \quad$ Maximum range of laser ranger

$\theta \quad$ Angle of intersection between two waypoint paths

\section{Introduction}

Autonomous miniature air vehicles (MAVs) have become popular lately. Their increasing ability to navigate intelligently and their low cost create opportunities for application in a wide range of fields. Their current use range from military intelligence and reconnaissance missions to domestic forest fire tracking. With the upward trend in MAV usage, we would like to make them more safe by avoiding possible obstacles and allowing them to navigate urban terrains using only light-weight hardware. Our goal in this paper is to derive an algorithm for obstacle avoidance for MAVs using only light-weight sensors.

Much work in obstacle avoidance in miniature air vehicles has been accomplished. One of the most noted ideas was the probability road map (PRM) developed in $^{2}$ and extended to non-holonomic systems in. ${ }^{3}$ In, ${ }^{3}$ the rapidly exploring random tree (RRT) for high dimensional spaces was developed. Assuming the locations of obstacles are known, an RRT will take random samples of the configuration space to use as possible waypoints to a goal, removing infeasible samples due to non-holonomic constraints. Each sample is selected and more random samples are taken to find additional waypoints. The result is a growing tree that

\footnotetext{
*Research Assistant, Electrical and Computer Engineering, Brigham Young University, Student Member AIAA.

$\dagger$ Associate Professor, Electrical and Computer Engineering, Brigham Young University, Senior Member AIAA.

¥Associate Professor, Mechanical Engineering, Brigham Young University, Senior Member AIAA.
} 
rapidly expands across a configuration space. In the presence of obstacles, an RRT can quickly find feasible paths around them. A nice extension for for the RRT in narrow passages was developed in. ${ }^{7}$ The RRT algorithm is excellent for path planning. In our case however, a good knowledge of the location of obstacles is not possible as is required in the RRT. We need a navigation algorithm incorporated with a sensor such that it may change paths dynamically as new information from the sensor is received.

To plan around obstacles they must first be detected, and detection is a problem for small aircraft. Most sensors capable of reliable obstacle detection in large areas, such as radar or laser scanners, are too heavy for small aircraft. One possible light weight sensor is a camera. Work has been done on using cameras and computer vision to detect obstacles. Cameras are light-weight and possible to mount on a MAV. In, ${ }^{5}$ vision is used to land a helicopter, i.e. ground detection. A 3D model is created from computer vision in ${ }^{1}$ and $^{6}$ which is used for navigation and obstacle avoidance. Computer vision can be used for obstacle avoidance using state of the art technology and algorithms. However, computer vision requires a large amount of computation which must be done on an off-board computer. We would like all of the computation to be self-contained onboard the MAV. Computer vision is not feasible for onboard computation.

We developed a dynamic algorithm for obstacle avoidance using a laser ranger in. ${ }^{4}$ The paper developed a method of creating temporary paths around obstacles as deviations from the waypoint path. However, the method gave no guarantee that the laser ranger would find obstacles near the waypoint path and no guarantee of obstacle avoidance. In this we paper we would like to present a method such that obstacle detection is guaranteed as well as obstacle avoidance.

Our goal is to incorporate a laser ranger sensor into an obstacle avoidance algorithm such that obstacle detection and obstacle avoidance are guaranteed. We assume each MAV is equipped with a laser ranger pointing in the $x$ direction of the body frame. A single laser ranger is light enough to mount on a MAV and allows ranging for obstacles directly in front of the MAV. We will derive a series of circular paths around a waypoint path such that the MAV may scan obstacles near the waypoint path and possible escape routes to avoid detected obstacles. Our goal is to guarantee obstacle detection and extend previously derived methods of obstacle avoidance to real time and account for frequent changes in the environment.

The organization of this paper is as follows. A new set of paths used for scanning and obstacle avoidance is introduced in section II. The path is analyzed and conditions for obstacle avoidance are described in section III. Section IV explores transitions between waypoint paths and section V summarizes the results.

\section{Circular Paths}

Our goal is to generate an oscillating path along which a MAV may traverse to scan potential obstacles. The desired path must conform the non-holonomic constraints of a fixed wing aircraft and enable the MAV to scan possible new paths ahead. Consider the first order set of kinematic equations of a fixed wing MAV,

$$
\begin{aligned}
\dot{r}_{n} & =V \cos \psi \\
\dot{r}_{e} & =V \sin \psi \\
\dot{\psi} & =\frac{g}{V} \tan \phi \\
\dot{V} & =\alpha_{V}\left(V^{c}-V\right) \\
\dot{\phi} & =\alpha_{\phi}\left(\phi^{c}-\phi\right),
\end{aligned}
$$

where $\left(r_{n}, r_{e}\right)^{T}$ is the position of the aircraft, $V, V_{c}, \phi$, and $\psi$ are the velocity, commanded velocity, roll, and heading, $g$ is the gravitational constant, and $\alpha_{\phi}$ and $\alpha_{V}$ are parameters. Using these equations, a constant roll angle $\phi_{d}$ results in a constant heading change $\dot{\psi}$. The aircraft flies a circular trajectory with a turning radius $R$. With a mounted laser ranger, the MAV scans nearby regions outside the circular path.

We would like to use the scanning abilities of the circular path by incorporating a series of circular oscillations in waypoint paths such that the MAV scans obstacles on or near a waypoint path and any possible escape routes that can be flown to avoid obstacles. Let $\psi_{d}$ be the desired heading of the MAV along a waypoint path, let $R$ be the desired turning radius of the circular paths, and let $\psi_{m}$ be the maximum deviation from $\psi_{d}$ in the circular oscillation as shown in figure 1. Let the circular paths be arranged in such a way that they intersect at exactly one point, and the angle of the tangent line at that point be $\psi_{m}$ from the desired heading $\psi_{d}$. We want the MAV to track one of the circular paths until it reaches the 


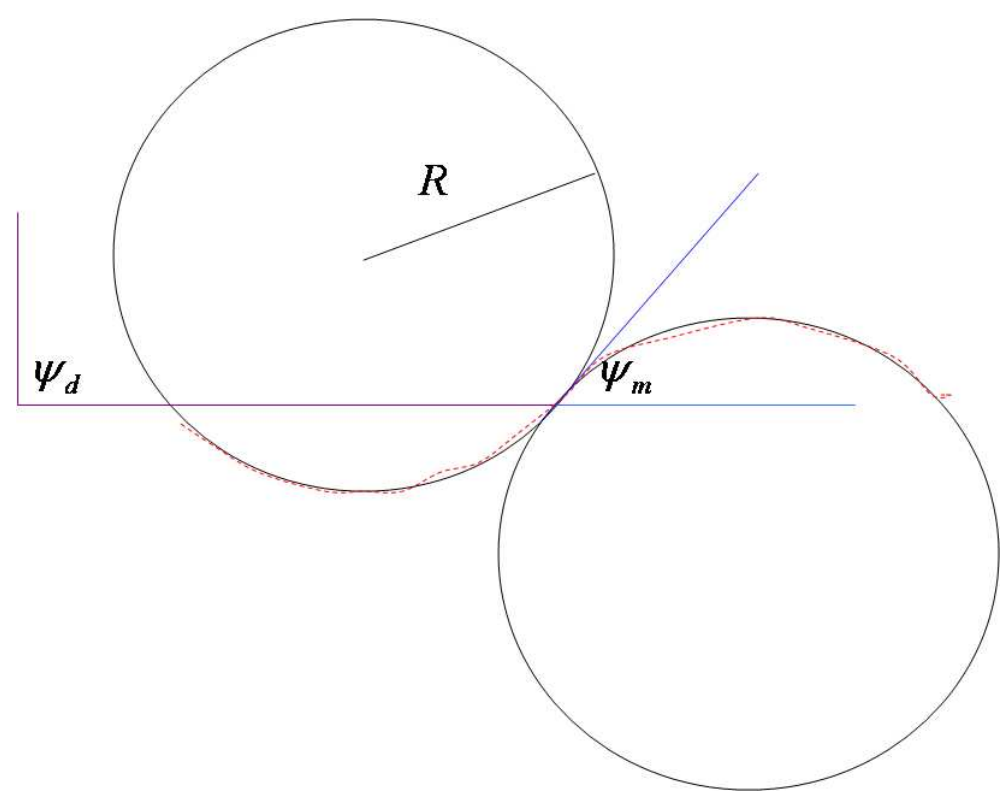

Figure 1. Geometry of a circular path around a waypoint path. The path of the MAV is shown in red.

intersection point of another circular path, it then begins to track the new circular path. The result is a series of oscillating circular paths around a waypoint path. We assume that the time to change roll angle is zero for simplicity.

\section{Analysis}

To analyze the conditions of the path that result in successful scanning, we need to know some of the properties of the path. Consider figure $2 . \overline{b d}$ is the desired path of travel. In other words, its orientation is $\psi_{d}$. The angle $\angle d b g$ is $\psi_{m}$ by definition. We can construct a line segment $\bar{f} g$ such that it is tangent to circles $a$ and $c$, passing through the point where the circles intersect at point $b . \bar{a} b$ is a radius of circle $a$ ending at point $b$, where the circle $a$ and $c$ intersect. $\overline{b c}$ is a radius of circle $c$ to point $b$. The line segment $\overline{a c}$ begins at the center of circle $a$ and ends at the edge of circle $c . \overline{a c}$ passes through point $b$ and is perpendicular to $\bar{f} g$. The length of $\overline{a c}$ is twice the radius of the circle, or $2 R$. Using complimentary angles, we know that $\angle c b d=\frac{\pi}{2}-\psi_{m} . \angle b c h=\psi_{m}$ using the sum of angles in a triangle. Using similar logic, we can find the other angles as labeled in figure 2 .

Using our knowledge of the angles, we find the lengths of the edges of the triangles in figure 2 . We know that $\ell(\overline{b h})=R \sin \left(\psi_{m}\right)$, and $\ell(\overline{c h})=R \cos \left(\psi_{m}\right)$, where $\ell(x)$ is the length of $x$. We can now find the distance from the center of one circle to the center of another circle. The distance from $a$ to $e$ is $4 R \sin \left(\psi_{m}\right)$. The distance from the center of a circle to a center $n$ circles ahead in the path is $2 n R \sin \left(\psi_{m}\right)$, where $n$ is an even number. In figure 2, the distance from $a$ to $e$ can be calculated using $n=2$, resulting in $4 R \sin \left(\psi_{m}\right)$ again.

To avoid obstacles successfully, the MAV needs to scan complete escape routes that may be flown in the case of obstacle detection. We will use the circles in the description of the path as possible escape paths from obstacles. These circles need to be scanned completely before the MAV starts traversing them to guarantee the escape route is obstacle free. We assume for a moment that $\psi_{d}$ does not change.

Theorem III.1. Let $\psi_{m}$ be the maximum deviation from a desired heading $\psi_{d}$ in a circular scanning path. Let $n$ be the number of circles ahead to scan and $L$ be the range of the laser ranger. If $\psi_{m}>\tan ^{-1}\left(\frac{\sqrt{n}}{n}\right)$ and $L \geq \sqrt{c^{2}+2 R c}$ where $c=2 R \sqrt{n^{2} \sin ^{2}\left(\psi_{m}\right)+\cos ^{2}\left(\psi_{m}\right)}$, then a circle $n$ circles ahead in the circular scanning path will be completely scanned.

Proof. Refer to figure 3(a). Let $\overline{a b}$ denote a tangent line of circles $e$ and $d$. Let $\theta$ be the angle of $\overline{a b}$ measured from $\psi_{d}$. This represents the laser ranger of the MAV. We need to verify the laser passes this location. Draw the radii $\overline{a e}$ and $\overline{b d}$ to the intersection points of the tangent line on circles $e$ and $d$ respectively. The 


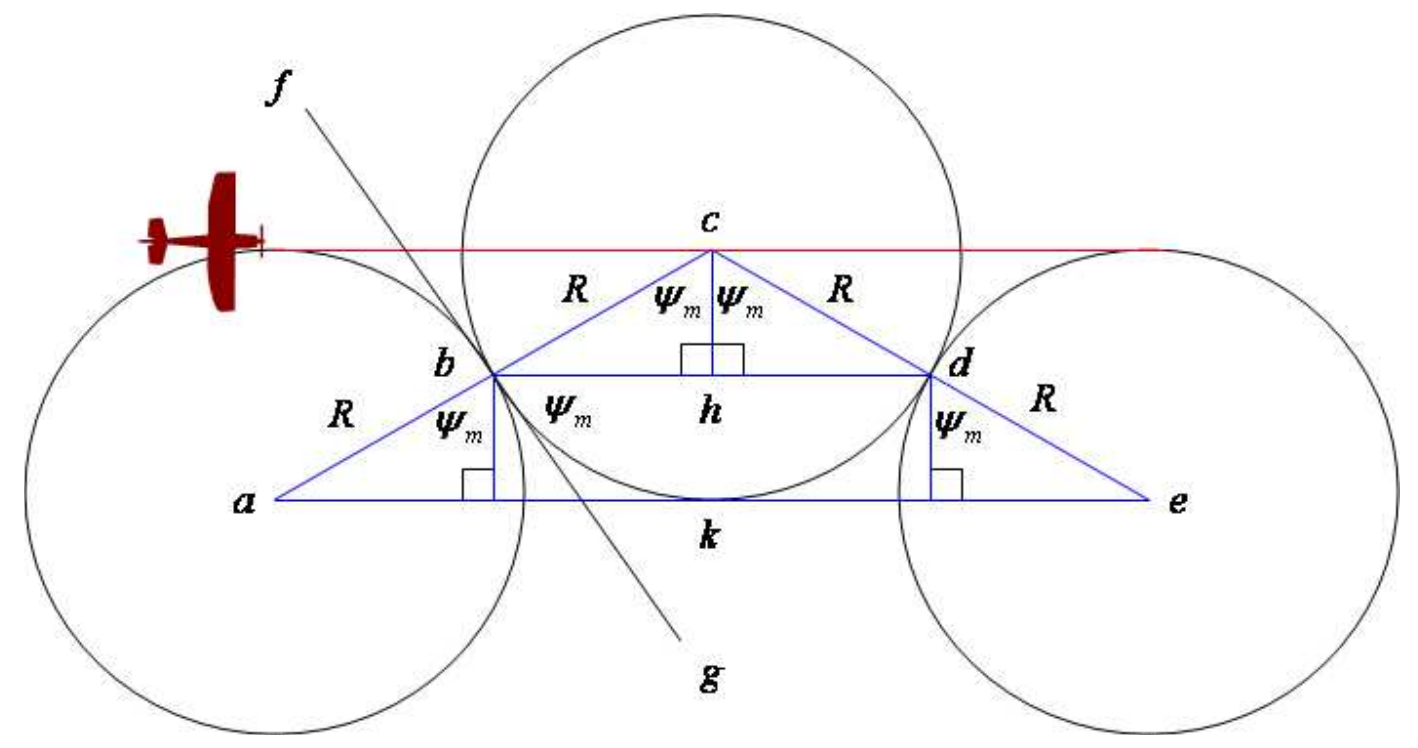

Figure 2. The measurements of the circular path can easily be found using geometry.

tangent line and radii are perpendicular to each other. Construct the line segment $\bar{e} d$ between the centers of the circles. This line is perpendicular to the radii and parallel to $\overline{a b} . \overline{a b}$ then has the same length and orientation as $\bar{e} d$. Construct the line segment $e^{-} f$ parallel to the direction of travel, and create the line segment $\overline{d f}$ perpendicular to it. $\angle d e f=\angle b a g$ because of the parallel lines $\overline{a b}, \overline{a g}$, éd, and $\bar{e} f$. $\angle e d f=\frac{\pi}{2}-\theta$ using the sum of triangle angles. Using the geometry discussed earlier, we know that $\ell(e f)=2 n R \sin \left(\psi_{m}\right)$ and $\ell(\overline{d f})=2 R \cos \left(\psi_{m}\right)$. The angle $\angle c a b=\frac{\pi}{2}-\theta$ by complimentary angles. We have shown that triangles $a b c$ and def have three equal angles and one equal side, therefore they are equivalent triangles. We can now use the lengths of the sides of triangle def on triangle $a b c$. An equation for the relation of $\theta$ and $\psi_{m}$ can be constructed as

$$
\frac{\pi}{2}-\theta=\tan ^{-1}\left(\frac{2 n R \sin \left(\psi_{m}\right)}{2 R \cos \left(\psi_{m}\right)}\right) .
$$

We want to know the angle where $\psi_{m}=\theta$, this is the smallest angle for $\psi_{m}$ to ensure the laser passes this tangent line. Substituting $\psi_{m}$ for $\theta$ and solving we get

$$
\psi_{m}=\tan ^{-1}\left(\frac{\sqrt{n}}{n}\right) .
$$

This is the angle at which the laser scans the edge of the circle. $\psi_{m}$ may be larger and still cross the tangent line, therefore

$$
\psi_{m} \geq \tan ^{-1}\left(\frac{\sqrt{n}}{n}\right)
$$

We know if $\psi_{m} \geq \tan ^{-1}\left(\frac{\sqrt{n}}{n}\right)$, then the laser ranger begins to scan circle $d$. We need to show the laser ranger completes the scan. Consider figure $3(\mathrm{~b}) \cdot \overline{a b}$ is a tangent line of circles $e$ and $d$ with $\overline{e a}$ and $\overline{d b}$ as radii of the circles to the tangent points. $\overline{a c}$ is parallel to the direction of travel (orientation $\psi_{d}$ ). $\alpha$ is the angle of the tangent line from the direction of travel. $\overline{a b}$ represents the orientation the laser ranger must achieve to completely scan circle $d$. We know that if $\psi_{m} \geq \alpha$, then circle $e$ will be completely scanned.

In triangle $a b c, \angle a c b$ is $\frac{\pi}{2}-\alpha$. This implies $\angle d c j=\frac{\pi}{2}-\alpha$. By the sums of angles in a triangle, $\angle c d j=\alpha$. The length $\ell(\bar{d} i)=2 R \cos \psi_{m}$ was derived above. Using the angle $\angle b d f$, we know $\ell(\overline{d f})=\ell(\bar{h})=R \cos \alpha$. 
Using the difference of $\ell(\overline{j i})$ and $\ell(\bar{d} i)$, we can find $\ell(\overline{d j})=2 R \cos \psi_{m}-R \cos \alpha$. Using $\ell(\bar{d} j)$, then $\ell(\bar{d} c)=$ $\frac{2 R \cos \psi_{m}-R \cos \alpha}{\cos \alpha} \cdot \ell(\overline{c b})$ is easily seen now as:

$$
\ell(\overline{c b})=R-\left(\frac{2 R \cos \psi_{m}-R \cos \alpha}{\cos \alpha}\right)=2 R\left(1-\frac{\cos \psi_{m}}{\cos \alpha}\right)
$$

Notice that in equation 9 we require that $\frac{\cos \psi_{m}}{\cos \alpha} \leq 1$ for the length to be positive. This implies $\cos \psi_{m} \leq$ $\cos \alpha$. Given triangle $a b c$, we know $0 \leq \alpha \leq \frac{\pi}{2}$. Given $0 \leq \psi_{m} \leq \pi$, then $\cos \psi_{m} \leq \cos \alpha \Rightarrow \psi_{m} \geq \alpha$. Thus the laser always achieves position $\overline{a b}$. If $\psi_{m} \geq \tan ^{-1}\left(\frac{\sqrt{n}}{n}\right)$, then circle $e$ is completely scanned.

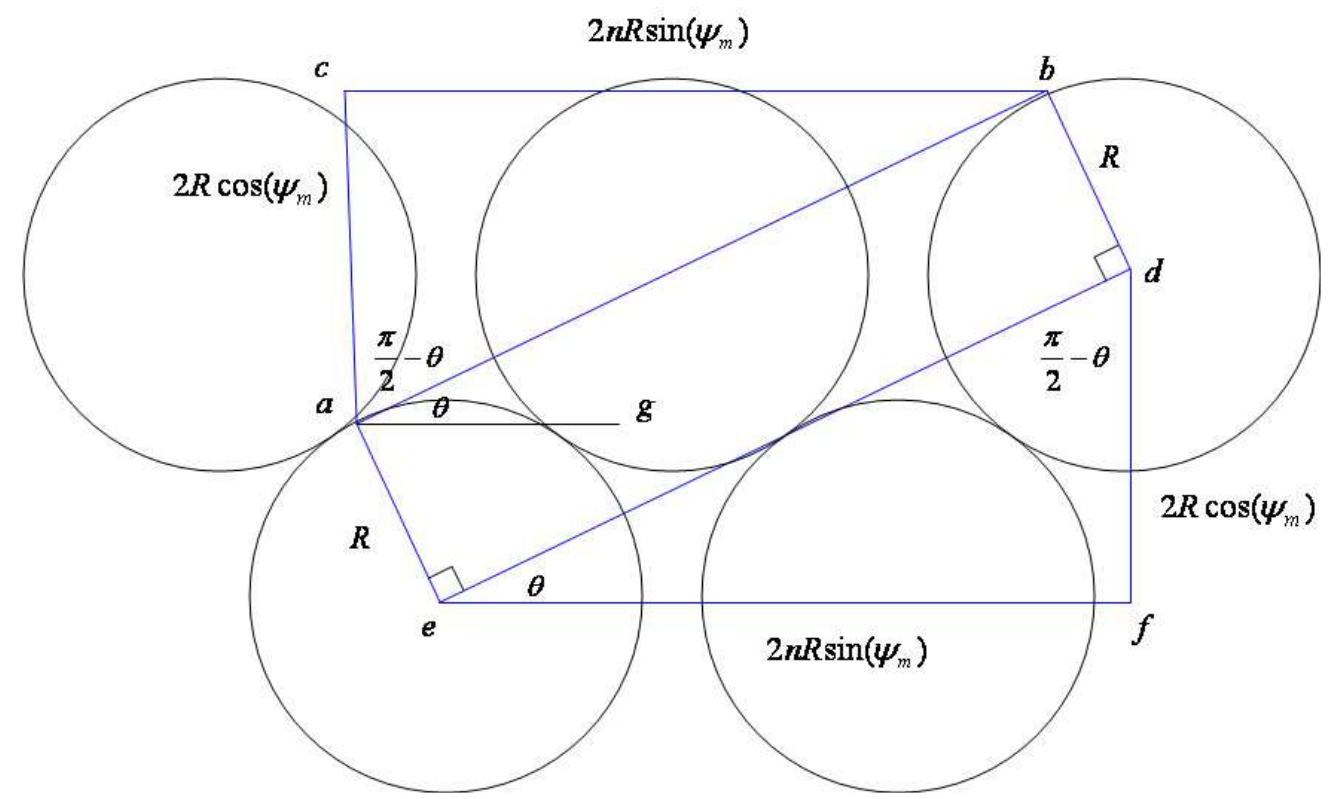

(a) $\overline{a b}$ represents the position the laser ranger must achieve to start scanning circle $e . \quad \psi_{m}>$ $\tan ^{-1}\left(\frac{\sqrt{n}}{n}\right)$ results in the laser ranger scanning a circular route $n$ circles ahead in the path.

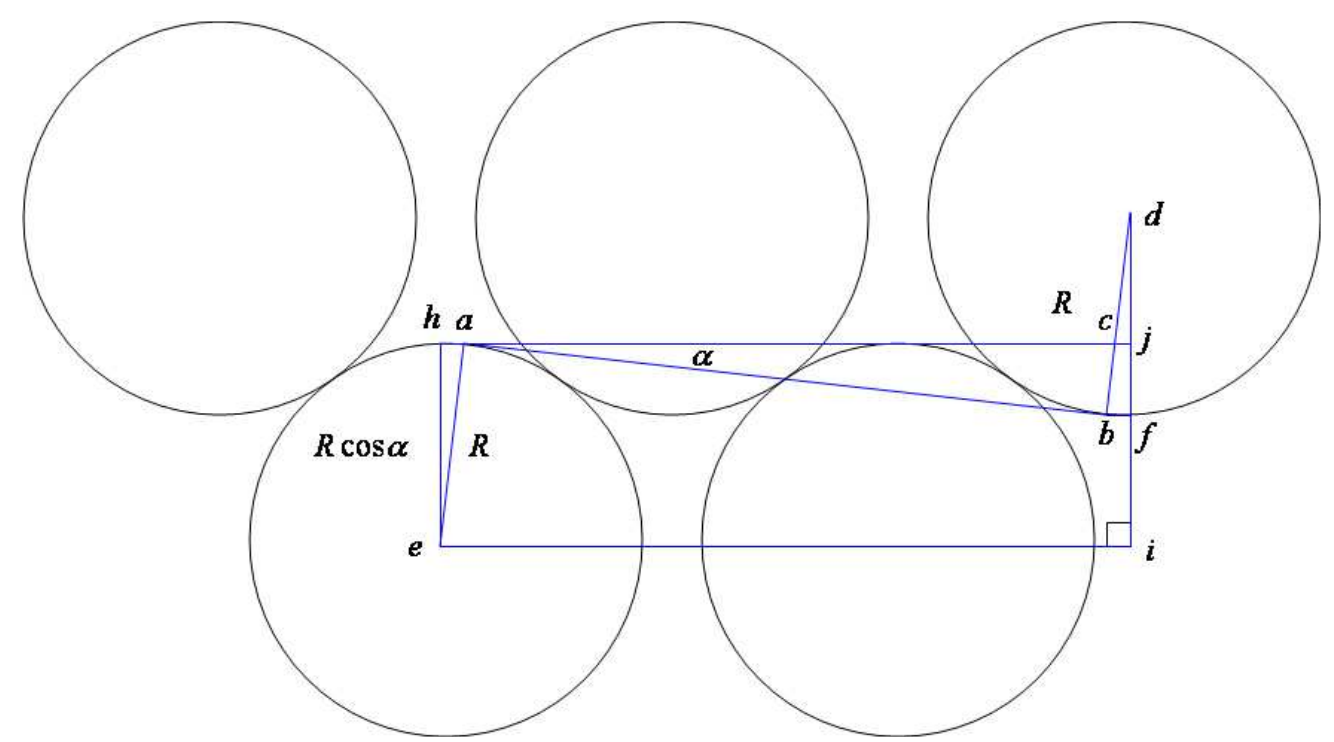

(b) $\overline{a b}$ represents the position the laser ranger must achieve to finish scanning circle $d$. We can find that $\alpha<\psi_{m}$ regardless of the chosen value of $\psi_{m}$.

Figure 3. Geometry used in derivations of the requirements of the circular path to guarantee scanned paths.

The next step is to determine a sufficient laser range to ensure a circular path is completely scanned. 
Consider figure 5(a). We can find the area the laser scans as the MAV tracks circle $a$. The area is a circle centered at $a$ with a radius of $\sqrt{R^{2}+L^{2}}$. Let circle $e$ be a circle we desire to scan $n$ circles ahead in the path. The distance from $a$ to the center of circle $e$ is

$$
\ell(\overline{a e})=\sqrt{\left(2 R n \sin \left(\psi_{m}\right)\right)^{2}+\left(2 R \cos \left(\psi_{m}\right)\right)^{2}} .
$$

The distance from $a$ to any point on circle $e$ has an upper bound of $\ell(\overline{a e})+R$. To guarantee that the laser has sufficient range to scan circle $e$, we can set the upper bound of the distance from $a$ to any point on circle $e$ equal to the radius of the circle scanned by the laser and solve for $L$,

$$
\sqrt{R^{2}+L^{2}} \geq \sqrt{\left(2 R n \sin \left(\psi_{m}\right)\right)^{2}+\left(2 R \cos \left(\psi_{m}\right)\right)^{2}}+R
$$

To simplify, let $c=\sqrt{\left(2 R n \sin \left(\psi_{m}\right)\right)^{2}+\left(2 R \cos \left(\psi_{m}\right)\right)^{2}}$. Then

$$
\sqrt{R^{2}+L^{2}} \geq c+R
$$

The required range of the laser to scan circle $e$ is

$$
L \geq \sqrt{c^{2}+2 R c}
$$

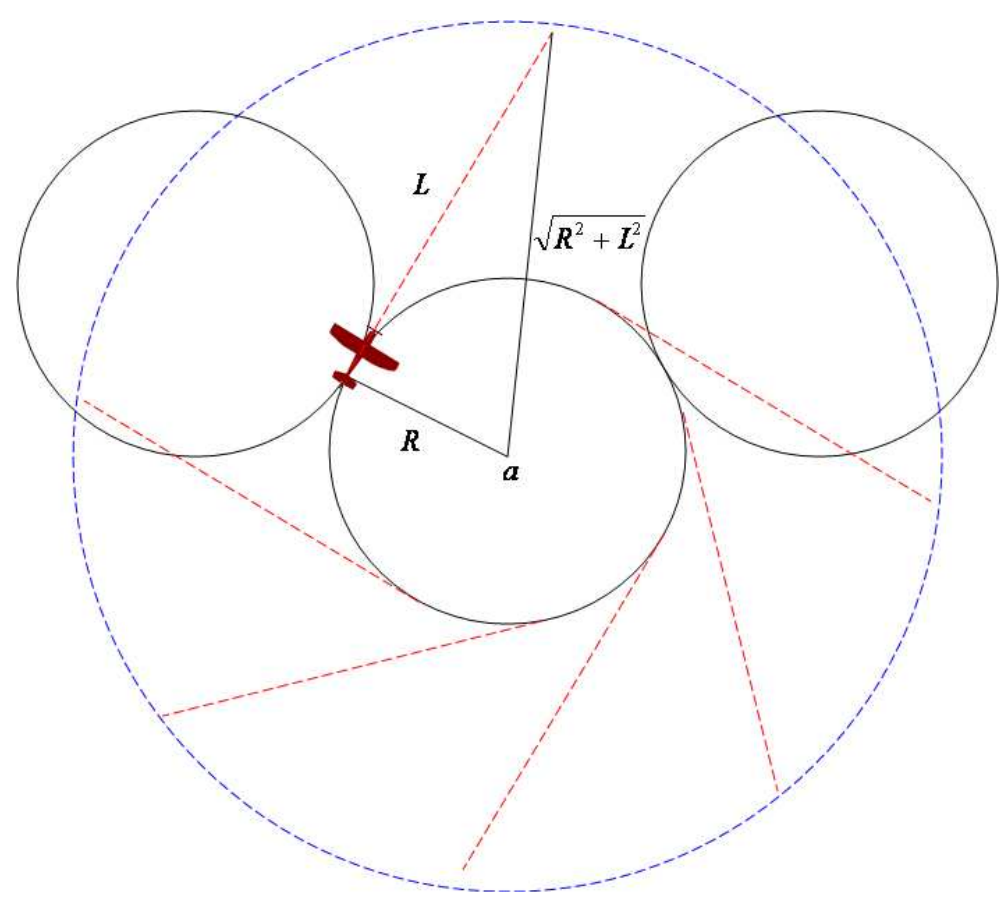

Figure 4. The blue circle is the area scanned by the laser as the MAV tracks circle $a$.

We have shown thus far the required conditions on the circular paths to guarantee obstacles will be detected before collision. In addition, these obstacles will be detected while tracking an obstacle free circle. In the case an obstacle is detected, two options are obvious. The first is to turn around using the circular orbit currently being flown and back track. The second is to fly the current circular orbit in it's full 360 degrees in an attempt to scan for a new obstacle free path in a different direction. Once another obstacle free path is found, the circular paths can continue in a new direction. Either method guarantees obstacle avoidance. 


\section{Waypoint Path Transitions}

An important part of flying waypoint paths is the transition between paths at waypoint junctions. We need to devise a method of smooth transition from one path to another. Consider figure 5(a). The red dashed line represents the direction of travel of a MAV. The green dashed line is a new waypoint path the MAV must transition to. As the MAV approaches the new path, it must change direction by tracking circles of its current path until reaching a tangent with a circle of the new path, after which it can track the new path. This is represented in the path from circle $a$ to circle $b$. We call the first circle of the new path the transition circle, shown as circle $b$.

The difficult part in the new path is finding the location of the transition circle. The derivation for finding the center of circle $b$ is illustrated in figure $5(\mathrm{~b})$. We want to find circle $b$ given the change in heading of $\theta$ and an intersection point of both paths with an offset of $(x, R \cos (\psi))$ from the center of circle $a$. Note that $x$ may be negative here if the offset is to the left. The following theorem shows how to find the location of the center of circle $b$.

Theorem IV.1. Let two waypoint paths intersect with angle $\theta$. Let circle a be the circle on the first waypoint path nearest the intersection point without crossing it. Let $x$ be the distance of a to the intersection point along the waypoint path. The location of the center of the transition circle is

$$
\begin{aligned}
& b_{x}=\frac{-(2 \tan \theta+2 q) \pm \sqrt{(2 \tan \theta+q)^{2}-4\left(\tan ^{2} \theta+1\right)\left(q^{2}-4 R^{2}\right)}}{2 \tan ^{2} \theta+2}, \\
& b_{y}=b_{x} \tan \theta+q,
\end{aligned}
$$

where

$$
q=\tan \theta\left(-x+R \cos \left(\frac{\pi}{2}-\theta+\psi_{m}\right)+\frac{R \sin \left(\frac{\pi}{2}-\theta+\psi_{m}\right)}{\tan \theta}\right)+R \cos \left(\psi_{m}\right)
$$

Proof. Refer to figure 5(b) for illustration of this proof. First translate circle $b$ along the new waypoint path $\bar{d} e$ such that the center of the circle lies on the first waypoint path $\bar{c} f$, this is circle $c$. Line segments $\overline{d f}$ and $\overline{e g}$ are tangent lines of circle $c$ at angles of $\psi_{m}$ along the direction of travel. $\bar{c} d$ and $\overline{c e}$ are radii of circle $c$. By opposite angles $\angle e d f=\angle d e g=\psi_{m}$. By adding the sum of the angles in a triangle, $\angle e g c=\theta-\psi_{m}$. Using the right triangle $\triangle c e g$ and summing the angles, we find $\angle e c g=\frac{\pi}{2}-\theta+\psi_{m}$. Construct the line segment $\overline{e h}$ perpendicular to $\bar{c} f$. Using the two right triangles formed, we can find the following,

$$
\begin{aligned}
\ell(\overline{c h}) & =R \cos \left(\frac{\pi}{2}-\theta+\psi_{m}\right) \\
\ell(\overline{e h}) & =R \sin \left(\frac{\pi}{2}-\theta+\psi_{m}\right) \\
\ell(\overline{h k}) & =\frac{R \sin \left(\frac{\pi}{2}-\theta+\psi_{m}\right)}{\tan \theta} \\
\ell(\bar{m} c) & =x-\ell(\overline{c h})-\ell(\overline{h k}) \\
& =x-R \cos \left(\frac{\pi}{2}-\theta+\psi_{m}\right)-\frac{R \sin \left(\frac{\pi}{2}-\theta+\psi_{m}\right)}{\tan \theta} \\
\ell(a \bar{m}) & =R \cos \left(\psi_{m}\right)
\end{aligned}
$$

Knowing the location of $c$, we can shift circle $c$ back along the waypoint path until it arrives at circle $b$, which can also be describes as when $\ell(\overline{a c})=2 R$. First create the equation of the line which crosses through $c$ and runs parallel to $\overline{d e}$,

$$
b_{y}=\tan \theta\left(b_{x}-x+R \cos \left(\frac{\pi}{2}-\theta+\psi_{m}\right)+\frac{R \sin \left(\frac{\pi}{2}-\theta+\psi_{m}\right)}{\tan \theta}\right)+R \cos \left(\psi_{m}\right) .
$$




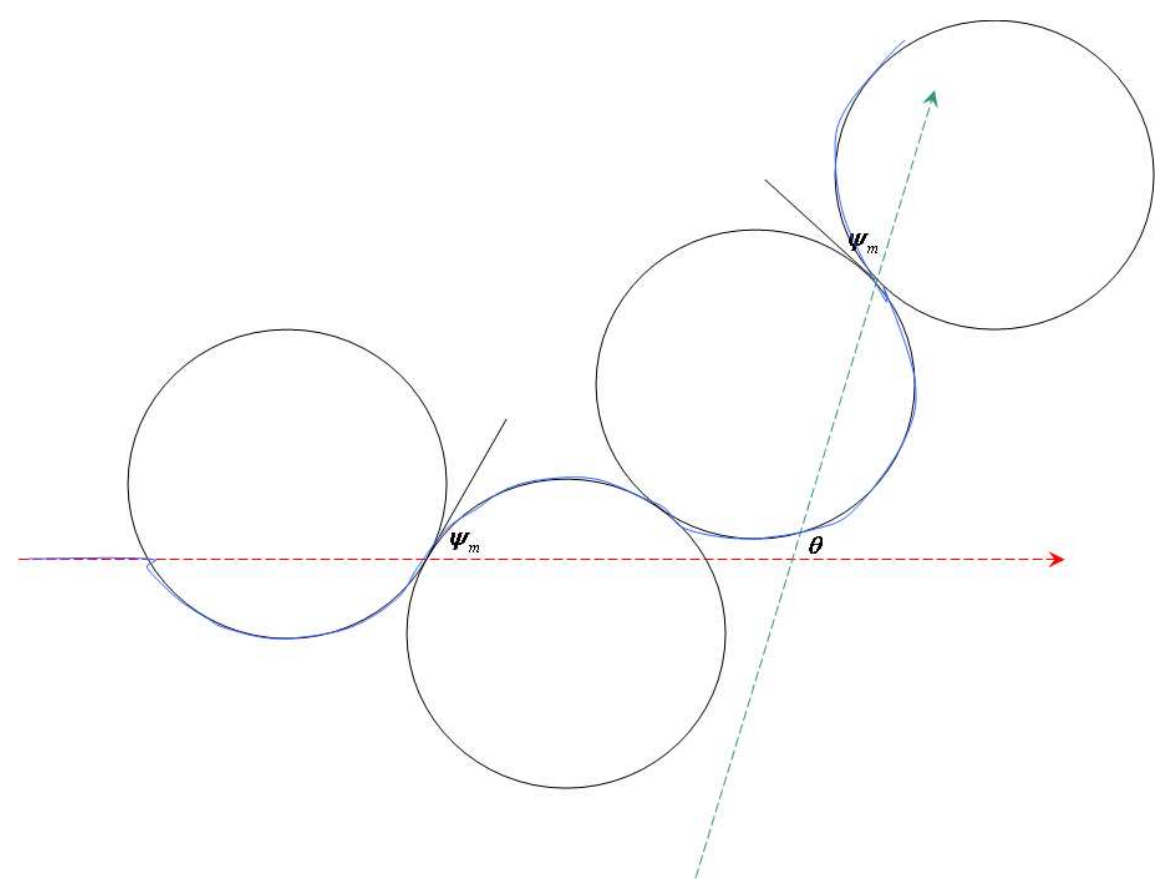

(a) Simple figure of a waypoint path transition is shown and the path a MAV flies through it.

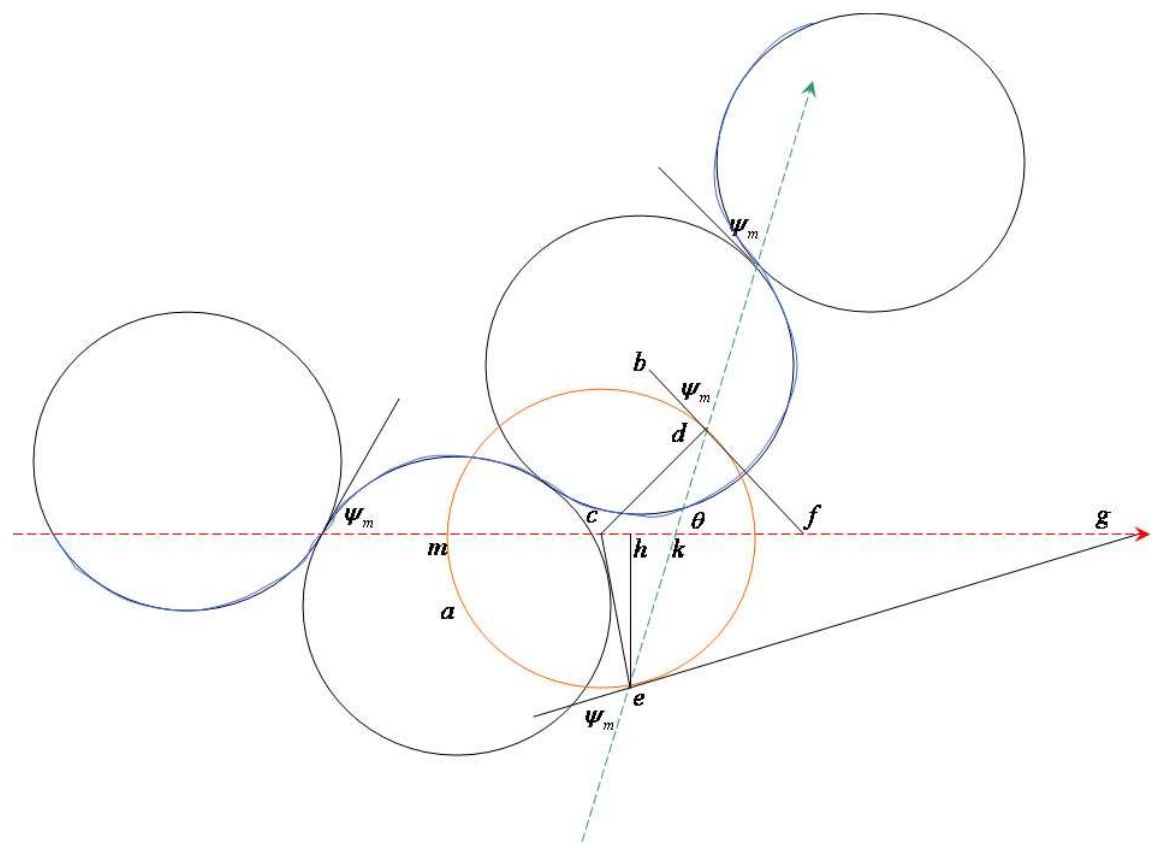

(b) Derivation for finding the center of the first circle on the new waypoint path.

Figure 5. The path of a MAV is shown during a waypoint change. The red dashed line represents the first waypoint path, and the green dashed line represents the second waypoint path. The blue line shows the path of a MAV flying the waypoint path transition. The goal is to find the center of circle $b$ which is the first circle for the MAV to track on the second waypoint path. 
Let

$$
q=\tan \theta\left(-x+R \cos \left(\frac{\pi}{2}-\theta+\psi_{m}\right)+\frac{R \sin \left(\frac{\pi}{2}-\theta+\psi_{m}\right)}{\tan \theta}\right)+R \cos \left(\psi_{m}\right)
$$

The equation can be simplified to

$$
b_{y}=b_{x} \tan \theta+q .
$$

We need to know the distance from $a$ to any point on this line, and find where that equals $2 R$. First set the distance equal to $2 R$ and solve for $b_{x}$,

$$
\begin{aligned}
2 R & =\sqrt{\left(b_{x} \tan \theta+q\right)^{2}+b_{x}} \\
4 R^{2} & =\left(\tan ^{2} \theta+1\right) b_{x}^{2}+2(\tan \theta+q) b_{x}+q^{2} \\
0 & =\left(\tan ^{2} \theta+1\right) b_{x}^{2}+2(\tan \theta+q) b_{x}+\left(q^{2}-4 R^{2}\right) \\
b_{x} & =\frac{-(2 \tan \theta+2 q) \pm \sqrt{(2 \tan \theta+q)^{2}-4\left(\tan ^{2} \theta+1\right)\left(q^{2}-4 R^{2}\right)}}{2 \tan ^{2} \theta+2}
\end{aligned}
$$

$b_{y}$ can be found by substituting $b_{x}$ back into equation 25 . The result is the location of circle $b$ as an offset from the center of circle $a$. Remember that these coordinates are in reference to point $a$ and the rotation of $\psi_{d}$. To move them to world coordinates, they must be shifted by a and rotated by $\psi_{d}-\frac{\pi}{2}$.

\section{Conclusion}

In this paper we have described a set circular paths that oscillate around a waypoint path for the purpose of scanning obstacles with a laser ranger. The paths scan obstacles as well as escape paths that may be utilized to prevent collision. When an obstacle is detected, a feasible path to avoid that obstacle is guaranteed. The feasible path is the circle the MAV is flying when the obstacle is detected. A method of transition between waypoint paths was also developed.

With the introduction of this new class of paths, obstacle detection is guaranteed. Methods of obstacle avoidance other than the rudimentary methods mentioned in this paper can be explored in future work. The laser ranger acquires data at real time and previously studied methods may be adapted to update paths based on new laser data. Future work may also explore 3D scanning, 3D obstacle avoidance, and terrain mapping using the laser ranger.

\section{References}

\footnotetext{
${ }^{1}$ Takeo Kanade, Omead Amidi, and Qifa Ke. Real-time and 3d vision for autonomous small and micro air vehicles. 43rd IEEE Conference on Decision and Control, pages 1655-1662, 2004.

${ }^{2}$ Lydia E. Kavraki, Petr Svestka, Jean-Claude Latombe, and Mark H. Overmars. Probabilistic roadmaps for path planning in high-dimensional configuration spaces. IEEE Transaction On Robotics and Automation, 12(4):556-580, August 1996.

${ }^{3}$ S.M. LaValle and Jr. Kuffner, J.J. Randomized kinodynamic planning. IEEE International Conference on Robotics and Automation, 1:473-479, May 1999.

${ }^{4}$ Jeffrey B. Saunders, Brandon Call, Andrew Curtis, Randal W. Beard, and Timothy W. McLain. Static and dynamic obstacle avoidance in miniature air vehicles. AIAA Infotech@Aerospace, September 2005.

${ }^{5}$ Courtney S. Sharp, Orriid Shakernia, and S. Shankar Sastry. A vision system for landing an unmanned aerial vehicle. Proceedings of the 2001 IEEE International Conference on Robotics; 8. Automation, pages 1720-1727, 2001.

${ }^{6}$ Bruno Sinopoli, Mario Micheli, Gianluca Donatot, and T. John Koo*. Vision based navigation for an unmanned aerial vehicle. Proceedings of the 2001 IEEE International Conference on Robotics and Automation, pages 1757-1764, 2001.

${ }^{7}$ Zheng Sun, David Hsu, Tingting Jiang, Hanna Kurniawati, and John H. Reif. Narrow passage sampling for probabilistic roadmap planning. IEEE TRANSACTIONS ON ROBOTICS, 21(6):1105-1115, December 2005.
} 